Cite this: Nanoscale, 2014, 6, 8509

Received 20th March 2014 Accepted 22nd May 2014

DOI: $10.1039 / c 4 n r 01538 j$

www.rsc.org/nanoscale

\section{Type-tunable amplified spontaneous emission from core-seeded CdSe/CdS nanorods controlled by exciton-exciton interaction $\dagger$}

\author{
Yusuf Kelestemur, $\$^{a}$ Ahmet Fatih Cihan, $\$ \$^{a}$ Burak Guzelturk ${ }^{a}$ \\ and Hilmi Volkan Demir ${ }^{\star a b}$
}

Type-tunable optical gain performance of core-seeded CdSe/CdS nanorods is studied via two-photon optical pumping. Controlling the exciton-exciton interaction by varying the core and shell size, blueshifted and red-shifted modes of amplified spontaneous emission are systematically demonstrated and their type attributions are verified by time-resolved emission kinetics.

Colloidal semiconductor nanocrystals have recently arisen as promising candidates to be utilized as active gain media for lasing applications. ${ }^{1,2}$ With their band gap tunability (via size and shape modifications), efficient band edge emission (even at room temperature), discrete band structure, and synthesis through facile wet-chemistry, it is possible to achieve colortunable, low-threshold, temperature insensitive and solution processed lasers from colloidal quantum dots (CQDs)..$^{2-6}$ However, there are various problems and challenges associated with CQD-based lasers. One way of achieving stimulated emission from the CQDs is through multi-exciton generation. When multiple excitons are formed, the Auger recombination (AR) process becomes more pronounced and inhibits optical gain in CQDs. ${ }^{7-9}$ In addition, high intensity optical pumping, which is required for stimulated emission, can photo-damage the sample and decrease the stability. ${ }^{10}$ To address these issues, CQDs having suppressed AR and exhibiting a higher absorption cross-section, which can increase the stability of CQDs by lowering the optical gain threshold, are strongly required.

\footnotetext{
${ }^{a}$ Department of Physics, Department of Electrical and Electronics Engineering, UNAM Institute of Materials Science and Nanotechnology, Bilkent University, TR-06800, Ankara, Turkey.E-mail: volkan@stanfordalumni.org

${ }^{b}$ Luminous! Center of Excellence for Semiconductor Lighting and Displays, School of Electrical and Electronic Engineering, School of Physical and Mathematical Sciences, Nanyang Technological University, Nanyang Avenue, Singapore 639798, Singapore $\dagger$ Electronic supplementary information (ESI) available. See DOI: $10.1039 / \mathrm{c} 4 \mathrm{nr} 01538 \mathrm{j}$

\$ These authors have contributed equally.

$\S$ Present address: Department of Electrical Engineering, Stanford University, Stanford, CA 94305, USA.
}

As a subclass of nanocrystals, core/shell nanorods (NRs) have been extensively considered for lasing applications and optical gain studies to overcome these problems with possibility of engineering their electronic structure for longer gain lifetime and increased absorption cross-section. ${ }^{11-14}$ From different varieties of core/shell NRs, core-seeded CdSe/CdS NRs have become quite attractive. With advances in the colloidal synthesis of CdSe/CdS core/shell NRs, it is possible to synthesize highly efficient and crystalline CdSe/CdS core/shell NRs having a narrow size distribution by finely controlling their shape and size. ${ }^{15,16}$ Furthermore, due to shallow band offset for electrons in the CdSe/CdS core/shell material system, a partial separation of electron and hole wavefunctions is observed which is known as the quasi type-II electronic structure that contributes to the suppression of Auger recombination, which is highly critical for achieving lasing. ${ }^{11,16-18}$ The other advantage of having a lower energy barrier for electrons in CdSe/CdS NRs is that, by simply changing their core and shell sizes, it is possible to tailor the electron and hole wavefunction overlap and tune CdSe/CdS NRs from type-I-like to quasi-type-II-like band alignment, which can be an important design consideration when engineering the gain/loss mechanisms in practical lasing systems. ${ }^{19-23}$ Moreover, due to the narrower band gap of CdS with respect to $\mathrm{ZnS}$, the absorption cross-section of CdSe/CdS core/shell NRs is greatly enhanced. ${ }^{13}$

With all of these aforementioned promises, in the nanocrystal lasing context, CdSe/CdS NRs have become one of the most heavily studied materials systems. ${ }^{18,24}$ Recently, singlemode, single-exciton and tunable laser emission on a silica microsphere ${ }^{25}$ and low-threshold amplified spontaneous emission (ASE) and lasing with two-photon pumping mechanism ${ }^{10}$ have been successfully demonstrated with CdSe/CdS core/shell NRs. Moreover, it was shown that nearly temperature-independent ASE can be possible with CdSe/CdS core/shell NRs ${ }^{26}$ and controllable transition of the ASE peak from the CdSe core region to the CdS shell region can be finely tuned by changing the length of the nanorod and the excitation intensity. ${ }^{21}$ In addition, the tunability of electron and hole wavefunction 
overlap in this materials system has also been discussed in the literature for both entangled photon generation ${ }^{27}$ and nanocrystal lasing applications. ${ }^{20}$ However, to the best of our knowledge, controlled shifting of the ASE peak with respect to the spontaneous emission in CdSe/CdS NRs through systematically changing the electronic property of the NRs has not been demonstrated. Here, as a result of the exciton-exciton interaction engineering via adjusting the core and the shell size, we show the electronic type tunability of the NR feature resulting in blue-shifting (type-II-like), and red-shifting (type-Ilike) ASE with respect to the spontaneous emission of NRs pumped with the two-photon absorption (TPA) optical pumping mechanism. We also verify the type attributions of different size NRs by carefully studying their time-resolved decay dynamics, where transition from type-I to type-II-like behavior indicated longer decay lifetimes as a consequence of the decreased electron and hole wavefunction overlap, and hence, a smaller oscillator strength.

In this study, we synthesized highly efficient and stable coreseeded CdSe/CdS NRs having different core sizes and rod lengths according to the recipe from the literature. ${ }^{15}$ As it can be seen from the TEM images (Fig. 1), they exhibit a highly crystalline structure with a narrow size distribution, which results in higher photoluminescence quantum efficiency $(>40 \%)$. Owing to a very small lattice mismatch between CdSe and CdS (3.9\%), epitaxial growth of CdS shell is achieved. Therefore, it is not easy to differentiate CdSe and CdS regions and confirm the growth of the CdS rod region without using strain analysis from the TEM images. However, the formation of the CdS rod on top of the CdSe core can be confirmed easily from the sharp increase in the absorption spectrum around the $400-500 \mathrm{~nm}$ region (Fig. 1), which corresponds to the band gap of wurtzite CdS. In addition, with the increase in rod length, this signature

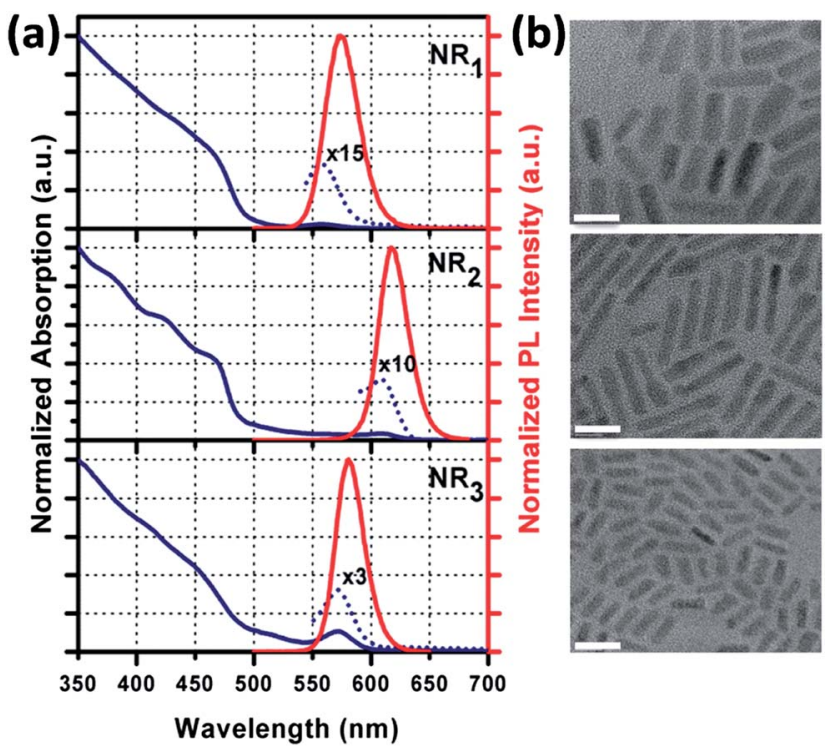

Fig. 1 (a) Absorption and photoluminescence spectra of CdSe/CdS core/shell NRs with varied core sizes and rod lengths and (b) their high resolution transmission electron microscopy (TEM) images (scale bars $=10 \mathrm{~nm}$ ). of the rod region on absorption becomes more dominant and makes a significant contribution to the absorption crosssection. Thanks to this very high absorption of mainly the rodshaped shell region of NRs, they are very good two-photon sensitizers, which is highly desirable for optical gain materials. $^{13}$

For the optical gain study, all three high quality close-packed films were prepared by drop casting of highly concentrated solutions (100 mg mL ${ }^{-1}$ ) of CdSe/CdS core/shell NRs on bare quartz substrates. The films were pumped with 120 fs laser pulses with a $1 \mathrm{kHz}$ repetition rate at the wavelength of $800 \mathrm{~nm}$ and the laser beam was focused on samples by using a cylindrical lens. The excitation intensity dependent emission spectrum of CdSe/CdS core/shell $\mathrm{NR}_{2}$ sample having a core diameter of 3.8-4.0 $\mathrm{nm}$ and a rod length of $30.0 \mathrm{~nm}$ is shown in Fig. 2. At low excitation intensities, only spontaneous emission was observed having a peak at $632 \mathrm{~nm}$ with a full-width-at-halfmaximum (FWHM) of $34 \mathrm{~nm}$. When the excitation intensity exceeds the threshold value $\left(\sim 7.5 \mathrm{~mJ} \mathrm{~cm}^{-2}\right)$, a slightly redshifted $(\sim 1 \mathrm{~nm})$ ASE peak emerged at $634 \mathrm{~nm}$ with a FWHM value of $7 \mathrm{~nm}$, which is very low as would be expected from an ASE process.

In addition, it was shown that due to the lower energy barrier for electrons in the CdSe/CdS core/shell material systems, a type tunable electronic structure from type-I to quasi type-II was achieved by changing the core size and rod length. ${ }^{22,30}$
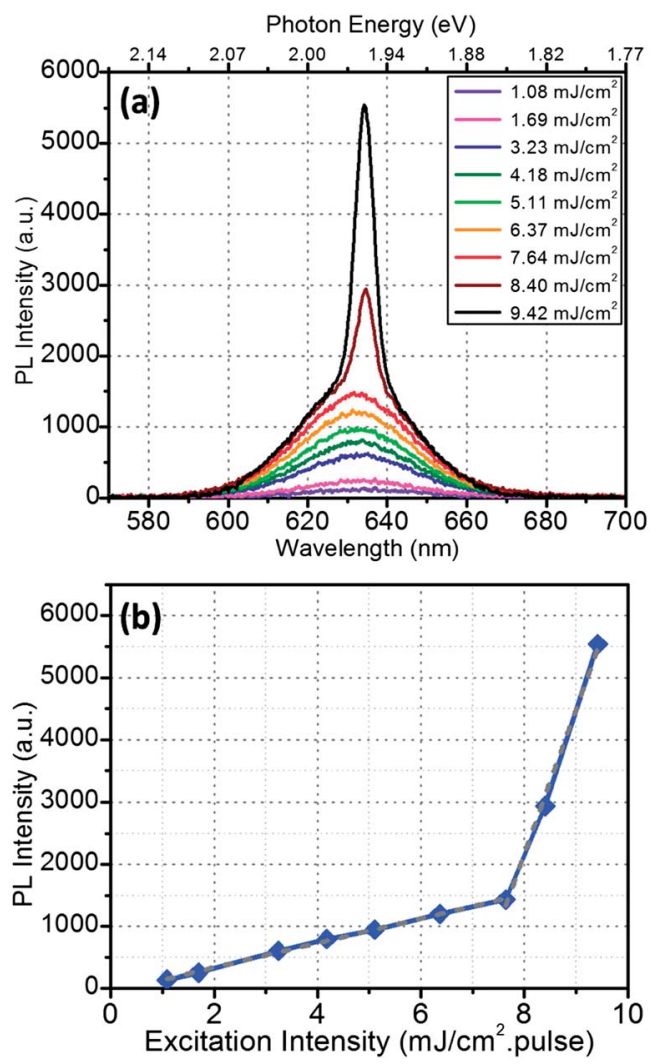

Fig. 2 (a) Photoluminescence emission spectra of a highly concentrated close-packed film of $\mathrm{CdSe} / \mathrm{CdS} \mathrm{NR}_{2}$ sample under different excitation pulse intensities and (b) excitation pulse intensity dependence of the emission at the ASE peak position of CdSe/CdS NR . 
Therefore, in order to figure out the effect of different electronic structures on optical gain performance from the same material system, we designed and synthesized CdSe/CdS NRs with different core sizes and shell thicknesses. The films were prepared by drop-casting of highly concentrated solution of CdSe/CdS NRs on bare quartz substrates having no loss compensation or waveguiding mechanism in order to make sure that the spectral position of the ASE peak is not affected by other parameters. We demonstrated type tunability of the ASE peak originating from biexciton emission with respect to spontaneous emission via engineering attractive $v s$. repulsive nature of exciton-exciton interactions in the NRs. As a result of the smaller core size of $\mathrm{NR}_{1}$, electrons were not confined to the CdSe core and a partial separation of electron and hole wavefunctions took place. Therefore, when we optically pumped the highly concentrated close-packed film of $\mathrm{NR}_{1}$, we observed typeII-like behavior with the approximately $9 \mathrm{~nm}$ blue-shifted ASE peak due to repulsive exciton-exciton interaction (Fig. 3). ${ }^{31,32}$ On the other hand, when the core size was increased as in the case of $\mathrm{NR}_{3}$, the leakage of electrons to the CdS shell decreased, meaning that the core could confine most of the electrons; therefore, type-I-like behavior was achieved with the $4 \mathrm{~nm}$ redshifted ASE peak from $\mathrm{NR}_{3}$ owing to attractive exciton-exciton interaction. Finally, we observed that attractive $v s$. repulsive nature of exciton-exciton interaction can be modified not only by changing the core size but also the rod length. As it can be seen from Fig. 3, the slightly red-shifted ( $\sim 1 \mathrm{~nm})$ ASE peak was achieved from the $\mathrm{NR}_{2}$ having a larger core size and a longer rod length, owing to the balanced attractive and repulsive excitonexciton interaction. For this sample, looking at only the core size one would suggest that the NRs should exhibit type-I-like behavior and looking at the shell length one would suggest that the NRs should behave like type-II. However, with the balanced dominances of these two localization regimes, which resulted in neither attractive nor repulsive exciton-exciton interaction, we achieved the slightly red-shifted ASE peak.

In order to verify the hypothesis on electron and hole wavefunction localization engineering stated above and gain more insight into the electronic band structure of CdSe/CdS core/ shell NRs with different core sizes and rod lengths, we conducted time-resolved fluorescence measurements (TRF). We performed TRF measurements by using samples of NRs in solution with low concentrations under lower excitation intensities to assure that the NRs are occupied with at most single excitons and nonradiative components are suppressed. TRF decay curves of CdSe/CdS NRs are shown in Fig. 4, and all of the

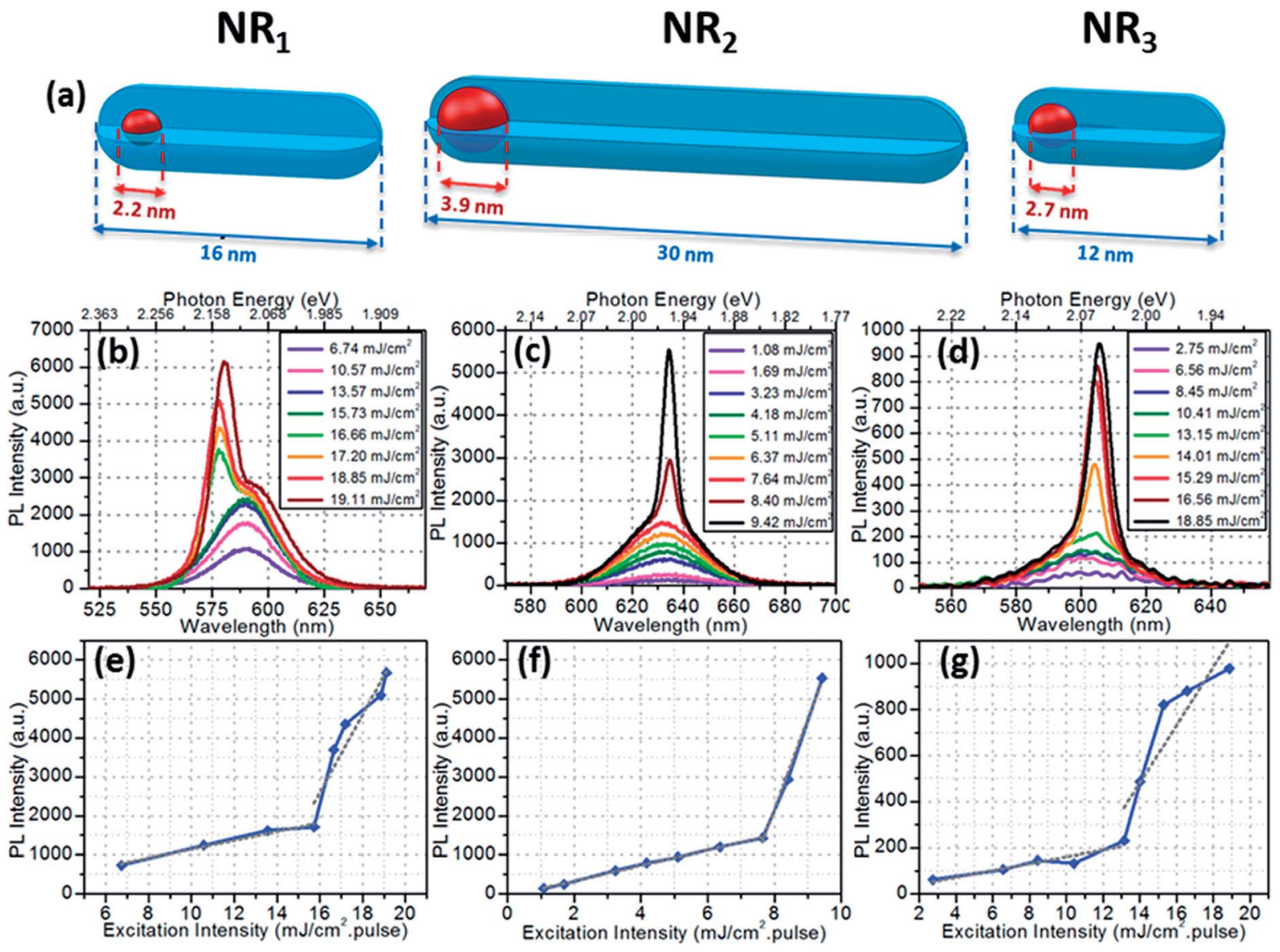

Fig. 3 (a) Schematic illustration of CdSe/CdS core/shell NRs proportional to their size. (b)-(d) Excitation intensity dependent emission spectra of $N R_{1}$ (with the blue-shifted ASE peak), NR $R_{2}$ (with the ASE peak at the same position as the spontaneous emission), and NR $R_{3}$ (with the red-shifted ASE peak), respectively. (e)-(g) Excitation pulse intensity dependences of emissions at the ASE peak positions of $N R_{1}, N_{2}$, and $N R_{3}$, respectively. 
decay curves exhibited almost single exponential decay. In the literature, for the bare CdSe core nanocrystals, decreased radiative lifetime was demonstrated with decreased core size and, for the CdSe/CdS core/shell NRs, increased lifetime was reported with increasing rod length. ${ }^{15}$ However, we observed the longest radiative lifetime ( $\sim 30.2 \mathrm{~ns})$ from the $\mathrm{NR}_{1}$ sample having the smaller core size and shorter rod length, which also matches with the observed type-II-like behavior from the blueshifted ASE behavior. ${ }^{33}$ In other words, owing to the smaller core size in $\mathrm{NR}_{1}$, electrons were delocalized over the CdS shell region and with the decreased wavefunction overlap of the electron and the hole, a longer radiative lifetime was observed (Fig. 4). On the other hand, with the increased core size, leakage of electrons to the shell region was reduced and shorter radiative lifetimes were measured from the $\mathrm{NR}_{3}$ as a result of increased electron and hole wavefunction overlap, which can be explained by the type-I-like behavior.

In order to perform a more detailed analysis on the tunability of the ASE peak, we also synthesized CdSe/CdS core/shell NRs having different rod lengths while the size of cores and the diameter of rods are kept the same. The size of cores and the diameter of rods are $3.9-4.1 \mathrm{~nm}$ and $5.0-6.5 \mathrm{~nm}$, respectively (Fig. S2 $\dagger$ ). Fig. 5 shows the photoluminescence spectra of CdSe/ CdS core/shell NRs having rod lengths of 22, 35 and $55 \mathrm{~nm}$ under intense two-photon optical pumping. In the case of $\mathrm{NR}_{4}$ having a shorter rod length, we achieved a $\sim 1 \mathrm{~nm}$ red-shifted ASE peak with a FHWM value of $7 \mathrm{~nm}$, which is expected due to their larger core size and shorter rod length (Fig. S3†). On the other hand, $8 \mathrm{~nm}$ blue-shifted ASE is observed for the case of $\mathrm{NR}_{5}$ having a longer rod, which can be explained with the decreased electron and hole wavefunction overlap as a result of the longer CdS rod region. This type-II-like ASE behavior of $\mathrm{NR}_{5}$ is also verified by using TRF measurements. As a result of the delocalization of electrons, a longer radiative lifetime (23.0 ns) is observed from $\mathrm{NR}_{5}$ while a decreased radiative lifetime (16.4 ns) is observed from $\mathrm{NR}_{4}$ (Fig. S4†). In addition, although $\mathrm{NR}_{6}$ has the longest CdS rod length, we achieved almost the same amount $(\sim 8 \mathrm{~nm})$ of blue-shifted ASE from $\mathrm{NR}_{6}$ when

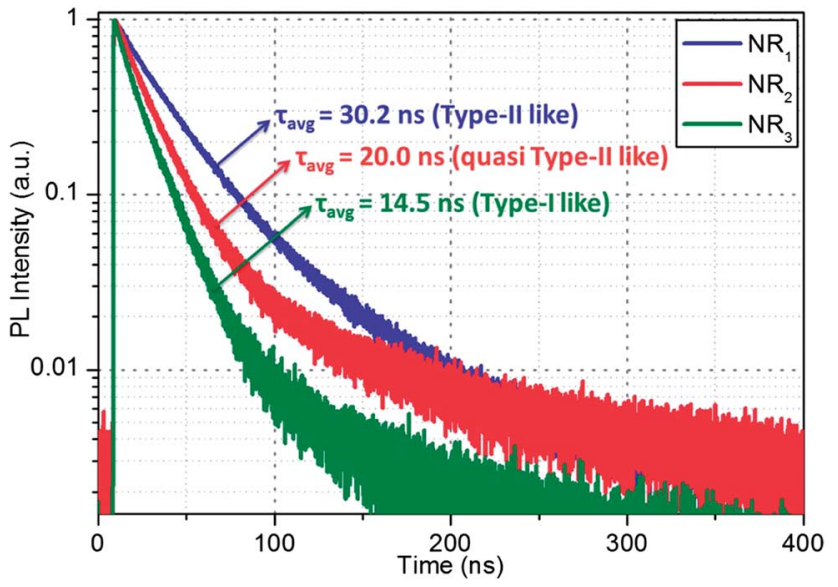

Fig. 4 TRF decay curves of the NRs together with average least chi-square fitting lifetimes. (a)

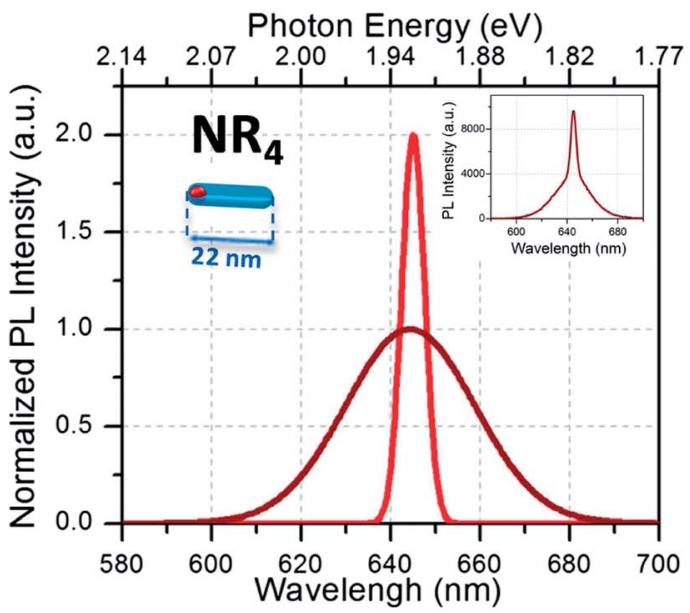

(b)

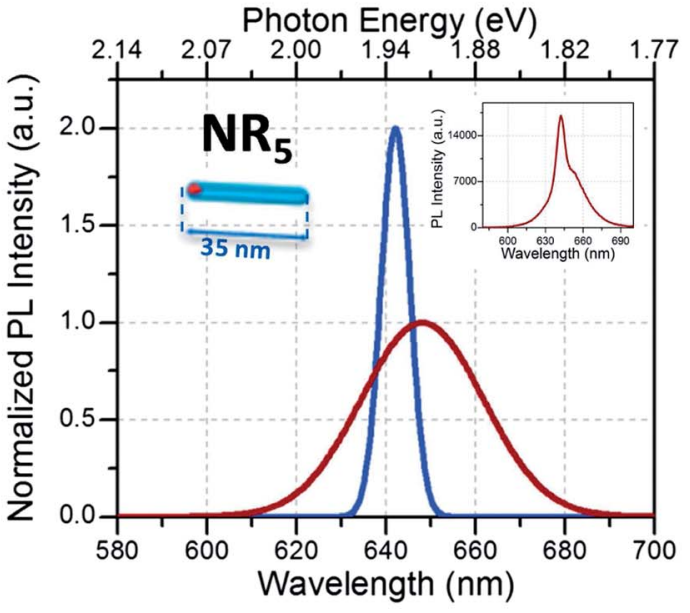

(c)

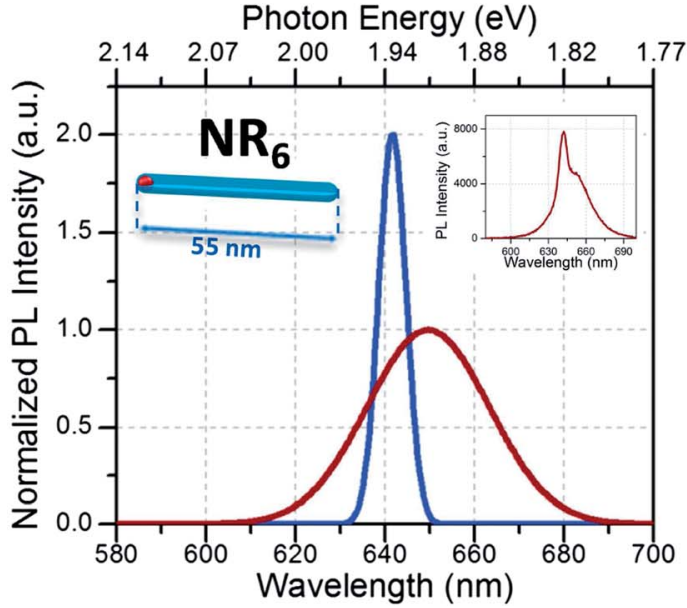

Fig. 5 Normalized decomposed ASE and spontaneous emission spectra of (a) $N R_{4}$, (b) $N R_{5}$ and (c) $N R_{6}$ samples under intense twophoton optical pumping. The broad spectra (normalized to unity) are the spontaneous emission spectra of the NRs, while the narrow spectra are the ASE spectra of the NRs. The experimental PL spectra of NRs without decomposition are demonstrated in the inset.

compared to $\mathrm{NR}_{5}$. It is also shown that both $\mathrm{NR}_{5}$ and $\mathrm{NR}_{6}$ have the same radiative lifetime, which confirms that photoluminescence lifetimes are a good indicator of confinement. Finally, optical gain thresholds down to $3 \mathrm{~mJ} \mathrm{~cm}^{-2}$ are achieved 
from red-emitting CdSe/CdS core/shell NRs. When compared to the previous studies of Jasieniak et al. $\left(7 \mathrm{~mJ} \mathrm{~cm}{ }^{-2}\right.$ threshold for red-emitting CdSe/CdS/ZnS CQDs employing a waveguiding mechanism $)^{28}$ and Todescato et al. $\left(12.31 \mathrm{~mJ} \mathrm{~cm}^{-2}\right.$ threshold for red-emitting CdSe/CdZnS/ZnS CQDs with a waveguiding mechanism) ${ }^{29}$ the optical gain threshold value can be said to be very low, which is attributed to the increased absorption crosssection.

\section{Conclusion}

In conclusion, we showed that with their giant two-photon absorption coefficients, CdSe/CdS NRs are one of the strongest candidates for lasing applications with two-photon optical pumping. Moreover, owing to their type tunable electronic configuration, blue-shifted and red-shifted ASE peaks with respect to spontaneous emission were achieved via engineering the exciton-exciton interaction in the $\mathrm{CdSe} / \mathrm{CdS}$ core/shell material system, which could prove useful when maximizing their optical gain performance, for example, through reducing reabsorption processes. Furthermore, we verified the type tunability of CdSe/CdS NRs with TRF measurements. These results show that with high two-photon absorption coefficient and ability to control the ASE peak shift, CdSe/CdS NRs are very promising candidates for practical lasing applications.

\section{Experimental section}

\section{Synthesis of CdSe/CdS core/shell nanorods}

CdSe/CdS core/shell NRs are synthesized by the seeded growth approach by using the slightly modified recipe from the literature. ${ }^{15}$ First, we synthesized CdSe cores. For a typical CdSe core synthesis, $3.0 \mathrm{~g}$ of trioctylphosphine oxide (TOPO), $0.280 \mathrm{~g}$ of octadecylphosphonic acid (ODPA) and $0.060 \mathrm{~g}$ of cadmium oxide (CdO) are loaded into a three-neck flask. The solution is evacuated at $150{ }^{\circ} \mathrm{C}$ for an hour to remove oxygen and water inside the reaction solution. Then, the solution is heated to $350{ }^{\circ} \mathrm{C}$ under an argon atmosphere for the dissolution of CdO. After the complete dissolution of $\mathrm{CdO}, 2 \mathrm{~mL}$ of trioctylphosphine (TOP) is injected swiftly. When the temperature is recovered to an injection temperature of $350{ }^{\circ} \mathrm{C}$, selenium (Se) stock solution $(0.058 \mathrm{~g}$ Se and $0.5 \mathrm{~mL}$ TOP) is injected. Different sizes of CdSe cores can be achieved by changing the growth time and the reaction is stopped by decreasing the temperature. Finally, CdSe cores are precipitated with ethanol and dissolved in toluene.

For the growth of CdS rods, $3 \mathrm{~g}$ of TOPO, $0.290 \mathrm{~g}$ of ODPA, $0.080 \mathrm{~g}$ of hexylphosphonic acid (HPA) and $0.060 \mathrm{~g}$ of CdO are loaded into the three-neck flask and evacuated at $150{ }^{\circ} \mathrm{C}$ for an hour. After evacuation, the temperature is increased to $350{ }^{\circ} \mathrm{C}$ for the dissolution of CdO under the argon atmosphere. When the solution becomes colorless, $2 \mathrm{~mL}$ of TOP is injected swiftly. After the temperature is recovered to $350{ }^{\circ} \mathrm{C}$, injection solution is injected and solution is kept at $350^{\circ} \mathrm{C}$ for the growth between 5 and $8 \mathrm{~min}$. For the preparation of injection solution, certain amounts of CdSe cores are precipitated. Then, these CdSe cores are dissolved in freshly prepared sulfur (s) stock solution $(0.200 \mathrm{~g} \mathrm{~S}$ and $2 \mathrm{~mL}$ TOP) inside the glove box. The reaction is stopped by decreasing the temperature and CdSe/CdS core/shell NRs are purified several times before optical gain studies by using ethanol. In addition, according to the desired rod length, the amounts of CdSe core, $\mathrm{CdO}$ and S can be tuned.

\section{Evaluation of the ASE spectra}

For the ASE experiments, as the excitation source for the NR samples, a Spectra Physics, Spitfire Pro XP regenerative amplifier having $120 \mathrm{fs}$ pulse width at $800 \mathrm{~nm}$ with a $1 \mathrm{kHz}$ repetition rate is used. The amplifier is seeded by a Ti:Sapphire laser (Spectra Physics, Tsunami). The laser beam is focused on the sample with the help of a cylindrical lens having a $20 \mathrm{~cm}$ focal length and the samples are excited with stripe geometry. A variable neutral density filter is used to adjust the pump intensity on the samples. The emission spectra of the samples were recorded by an optical fiber connected to a miniature spectrometer (Maya2000 Pro).

\section{Acknowledgements}

This work is supported in part by the Singapore National Research Foundation under the program numbers of NRFCRP6-2010-02 and NRF-RF-2009-09, and in part by ESF EURYI. Also, H.V.D. gratefully acknowledges support from TUBA-GEBIP. A.F.C. and Y.K. acknowledge support from TUBITAK BIDEB.

\section{References}

1 V. I. Klimov, A. A. Mikhailovsky, S. Xu, A. Malko, J. A. Hollingsworth, C. A. Leatherdale, H.-J. Eisler and M. G. Bawendi, Science, 2000, 290, 314-317.

2 C. Dang, J. Lee, C. Breen, J. S. Steckel and S. Coe-Sullivan, Nat. Nanotechnol., 2012, 7, 335-339.

3 C. B. Murray, D. J. Norris and M. G. Bawendi, J. Am. Chem. Soc., 1993, 115, 8706-8715.

4 M. Kazes, D. Y. Lewis, Y. Ebenstein, T. Mokari and U. Banin, Adv. Mater., 2002, 14, 317-321.

5 A. V. Malko, A. A. Mikhailovsky, M. A. Petruska, J. A. Hollingsworth, H. Htoon, M. G. Bawendi and V. I. Klimov, Appl. Phys. Lett., 2002, 81, 1303-1305.

6 X. Peng, M. C. Schlamp, A. V. Kadavanich and A. P. Alivisatos, J. Am. Chem. Soc., 1997, 119, 7019-7029.

7 V. I. Klimov, J. A. McGuire, R. D. Schaller and V. I. Rupasov, Phys. Rev. B: Condens. Matter Mater. Phys., 2008, 77, 195324195412.

8 B. Fisher, J. M. Caruge, Y. T. Chan, J. Halpert and M. G. Bawendi, Chem. Phys., 2005, 318, 71-81.

9 J. M. Caruge, Y. Chan, V. Sundar, H. J. Eisler and M. G. Bawendi, Phys. Rev. B: Condens. Matter Mater. Phys., 2004, 70, 085316-085317.

10 G. Xing, Y. Liao, X. Wu, S. Chakrabortty, X. Liu, E. K. L. Yeow, Y. Chan and T. C. Sum, ACS Nano, 2012, 6, 10835-10844.

11 M. Zavelani-Rossi, M. G. Lupo, F. Tassone, L. Manna and G. Lanzani, Nano Lett., 2010, 10, 3142-3150.

12 H. Htoon, J. A. Hollingworth, A. V. Malko, R. Dickerson and V. I. Klimov, Appl. Phys. Lett., 2003, 82, 4776-4778. 
13 G. Xing, S. Chakrabortty, K. L. Chou, N. Mishra, C. H. A. Huan, Y. Chan and T. C. Sum, Appl. Phys. Lett., 2010, 97, 061112-061113.

14 M. Zavelani-Rossi, M. G. Lupo, R. Krahne, L. Manna and G. Lanzani, Nanoscale, 2010, 2, 931-935.

15 L. Carbone, C. Nobile, M. De Giorgi, F. D. Sala, G. Morello, P. Pompa, M. Hytch, E. Snoeck, A. Fiore, I. R. Franchini, M. Nadasan, A. F. Silvestre, L. Chiodo, S. Kudera, R. Cingolani, R. Krahne and L. Manna, Nano Lett., 2007, 7, 2942-2950.

16 D. V. Talapin, R. Koeppe, S. Götzinger, A. Kornowski, J. M. Lupton, A. L. Rogach, O. Benson, J. Feldmann and H. Weller, Nano Lett., 2003, 3, 1677-1681.

17 F. García-Santamaría, S. Brovelli, R. Viswanatha, J. A. Hollingsworth, H. Htoon, S. A. Crooker and V. I. Klimov, Nano Lett., 2011, 11, 687-693.

18 F. García-Santamaría, Y. Chen, J. Vela, R. D. Schaller, J. A. Hollingsworth and V. I. Klimov, Nano Lett., 2009, 9, 3482-3488.

19 A. F. Cihan, P. L. H. Martinez, Y. Kelestemur, E. Mutlugun and H. V. Demir, ACS Nano, 2013, 7, 4799-4809.

20 M. Saba, S. Minniberger, F. Quochi, J. Roither, M. Marceddu, A. Gocalinska, M. V. Kovalenko, D. V. Talapin, W. Heiss, A. Mura and G. Bongiovanni, Adv. Mater., 2009, 21, 49424946.

21 R. Krahne, M. Zavelani-Rossi, M. G. Lupo, L. Manna and G. Lanzani, Appl. Phys. Lett., 2011, 98, 063105-063113.

22 A. Sitt, F. D. Sala, G. Menagen and U. Banin, Nano Lett., 2009, 9, 3470-3476.
23 A. F. Cihan, Y. Kelestemur, B. Guzelturk, O. Yerli, U. Kurum, H. G. Yaglioglu, A. Elmali and H. V. Demir, J. Phys. Chem. Lett., 2013, 4, 4146-4152.

24 Y. Liao, G. Xing, N. Mishra, T. C. Sum and Y. Chan, Adv. Mater., 2012, 24, OP159-164.

25 C. Grivas, C. Li, P. Andreakou, P. Wang, M. Ding, G. Brambilla, L. Manna and P. Lagoudakis, Nat. Commun., 2013, 4, 1-9.

26 I. Moreels, G. Rainò, R. Gomes, Z. Hens, T. Stöferle and R. F. Mahrt, Adv. Mater., 2012, 24, OP231-235.

27 G. Rainò, T. Stöferle, I. Moreels, R. Gomes, Z. Hens and R. F. Mahrt, ACS Nano, 2012, 6, 1979-1987.

28 J. J. Jasieniak, I. Fortunati, S. Gardin, R. Signorini, R. Bozio, A. Martucci and P. Mulvaney, Adv. Mater., 2008, 20, 69-73.

29 F. Todescato, I. Fortunati, S. Gardin, E. Garbin, E. Collini, R. Bozio, J. J. Jasieniak, G. D. Giustina, G. Brusatin, S. Toffanin and R. Signorini, Adv. Funct. Mater., 2012, 22, 337-344.

30 J. Müller, J. M. Lupton, P. G. Lagoudakis, F. Schindler, R. Koeppe, A. L. Rogach, J. Feldmann, D. V. Talapin and H. Weller, Nano Lett., 2005, 5, 2044-2049.

31 V. I. Klimov, S. A. Ivanov, J. Nanda, M. Achermann, I. Bezel, J. A. McGuire and A. Piryatinski, Nature, 2007, 447, 441446.

32 J. Nanda, S. A. Ivanov, M. Achermann, I. Bezel, A. Piryatinski and V. I. Klimov, J. Phys. Chem. C, 2007, 111, 1538215390.

33 G. Rainò, T. Stöferle, I. Moreels, R. Gomes, J. S. Kamal, Z. Hens and R. F. Mahrt, ACS Nano, 2011, 5, 4031-4036. 